\title{
Revisiting Galactic Rotation Curves Given a Noncommutative-Geometry Background
}

\author{
Peter K. F. Kuhfittig, Vance D. Gladney \\ Department of Mathematics, Milwaukee School of Engineering, Milwaukee, USA \\ Email: kuhfitti@msoe.edu
}

Received 5 September 2014; revised 2 October 2014; accepted 21 October 2014

Copyright (C) 2014 by authors and Scientific Research Publishing Inc.

This work is licensed under the Creative Commons Attribution International License (CC BY). http://creativecommons.org/licenses/by/4.0/

c) (i) Open Access

\begin{abstract}
It was shown earlier by Rahaman et al. that a noncommutative-geometry background can account for galactic rotation curves without the need for dark matter. The smearing effect that characterizes noncommutative geometry is described by means of a Gaussian distribution intended to replace the Dirac delta function. The purpose of this paper is two-fold: 1) to account for the galactic rotation curves in a more transparent and intuitively more appealing way by replacing the Gaussian function by the simpler Lorentzian distribution proposed by Nozari and Mehdipour and 2) to show that the smearing effect is both a necessary and sufficient condition for meeting the stability criterion.
\end{abstract}

\section{Keywords}

Galactic Rotation Curves, Noncommutative Geometry

\section{Introduction}

That noncommutative geometry can account for galactic rotation curves without the need for dark matter has already been shown in Ref. [1]. The effect in question is a small effect, not only difficult to determine but also difficult to present in an intuitively appealing way. This paper uses a slightly different approach that may provide a clearer picture. This approach is introduced in Section 2 followed by the analysis in Section 3. It is shown in Section 4 that the noncommutative-geometry background is needed for stability.

\section{Noncommutative Geometry and Galactic Rotation Curves}

An important outcome of string theory is the realization that coordinates may become noncommuting operators on a $D$-brane [2] [3]. The commutator is $\left[x^{\mu}, x^{v}\right]=i \theta^{\mu v}$, where $\theta^{\mu v}$ is an antisymmetric matrix. As discussed in Refs. [4] [5], noncommutativity reptaces point-like structures by smeared objects. The smearing effect 
is accomplished by using a Gaussian distribution of minimal length $\sqrt{\theta}$ instead of the Dirac delta function [6] [7]. A simpler but equally effective way is to assume that the energy density of the static and spherically symmetric and particle-like gravitational source has the form [8] [9]

$$
\rho(r)=\frac{M \sqrt{\theta}}{\pi^{2}\left(r^{2}+\theta\right)^{2}}
$$

Here the mass $M$ of the particle is diffused throughout the region of linear dimension $\sqrt{\theta}$ due to the uncertainty. The noncommutative geometry is an intrinsic property of spacetime and does not depend on any particular feature such as curvature.

To connect the noncommutative geometry to dark matter and hence to galactic rotation curves, we need to introduce the metric for a static spherically symmetric spacetime:

$$
\mathrm{d} s^{2}=-\mathrm{e}^{v(r)} \mathrm{d} t^{2}+\mathrm{e}^{\lambda(r)} \mathrm{d} r^{2}+r^{2}\left(\mathrm{~d} \theta^{2}+\sin ^{2} \theta \mathrm{d} \phi^{2}\right)
$$

For this metric, the Einstein field equations are

$$
\begin{aligned}
& \mathrm{e}^{-\lambda}\left[\frac{\lambda^{\prime}}{r}-\frac{1}{r^{2}}\right]+\frac{1}{r^{2}}=8 \pi \rho \\
& \mathrm{e}^{-\lambda}\left[\frac{1}{r^{2}}+\frac{v^{\prime}}{r}\right]-\frac{1}{r^{2}}=8 \pi p_{r}
\end{aligned}
$$

and

$$
\frac{1}{2} \mathrm{e}^{-\lambda}\left[\frac{1}{2}\left(v^{\prime}\right)^{2}+v^{\prime \prime}-\frac{1}{2} \lambda^{\prime} v^{\prime}+\frac{1}{r}\left(v^{\prime}-\lambda^{\prime}\right)\right]=8 \pi p_{t}
$$

One goal of any modified gravitational theory is to explain the peculiar behavior of galactic rotation curves without postulating the existence of dark matter: test particles move with constant tangential velocity $v^{\phi}$ in a circular path. It is noted in Ref. [10] that galactic rotation curves generally show much more complicated dynamics. For present purposes, however, the analysis can be restricted to the region in which the velocity is indeed constant. So taking the observed flat rotation curves as input, it is well known that, as a result,

$$
\mathrm{e}^{v}=B_{0} r^{l}
$$

where $l=2 v^{2 \phi}$ and $B_{0}$ is an integration constant [11]. Moreover, it is shown in Ref. [12] that in the presumed dark matter dominated region, $v^{\phi} \sim 300 \mathrm{~km} / \mathrm{s}=10^{-3}$ for a typical galaxy. So $l=0.000001$ [13] (we are using units in which $c=G=1$ ).

To address the issue of stable orbits, we first note that given the four-velocity $U^{\alpha}=\mathrm{d} x^{\alpha} / \mathrm{d} \tau$ of a test particle moving solely in the "equatorial plane" $\theta=\pi / 2$ of the galactic halo, the equation $g_{v \sigma} U^{v} U^{\sigma}=-m_{0}^{2}$ can be cast in the Newtonian form

$$
\left(\frac{\mathrm{d} r}{\mathrm{~d} \tau}\right)^{2}=E^{2}+V(r)
$$

which results in

$$
V(r)=-E^{2}+E^{2} \frac{\mathrm{e}^{-\lambda}}{B_{0} r^{l}}-\mathrm{e}^{-\lambda}\left(1+\frac{L^{2}}{r^{2}}\right)
$$

Here the constants $E$ and $L$ are, respectively, the conserved relativistic energy and angular momentum per unit rest mass of the test particle [13]. We are going to define circular orbits by $r=R_{0}$, a constant. We now have

$$
\frac{\mathrm{d} R_{0}}{\mathrm{~d} \tau}=0 \quad \text { and }\left.\quad \frac{\mathrm{d} V}{\mathrm{~d} r}\right|_{r=R_{0}}=0
$$


From these conditions, we obtain [1]

$$
L= \pm \sqrt{\frac{l}{2-l}} R_{0} \quad \text { and } \quad E= \pm \sqrt{\frac{2 B_{0}}{2-l}} R_{0}^{l / 2}
$$

The orbits are stable if

$$
\left.\frac{\mathrm{d}^{2} V}{\mathrm{~d} r^{2}}\right|_{r=R_{0}}<0
$$

and unstable if

$$
\left.\frac{\mathrm{d}^{2} V}{\mathrm{~d} r^{2}}\right|_{r=R_{0}}>0
$$

\section{The Solution}

The smeared gravitational source in Equation (1) leads to a smeared mass. More precisely, the Schwarzschild solution of the Einstein field equations associated with the smeared source leads to the line element

$$
\mathrm{d} s^{2}=-\left(1-\frac{2 M_{\theta}(r)}{r}\right) \mathrm{d} t^{2}+\left(1-\frac{2 M_{\theta}(r)}{r}\right)^{-1} \mathrm{~d} r^{2}+r^{2}\left(\mathrm{~d} \theta^{2}+\sin ^{2} \theta \mathrm{d} \phi^{2}\right)
$$

The smeared mass is implicitly given by

$$
M_{\theta}(r)=\int_{0}^{r} \rho\left(r^{\prime}\right) 4 \pi\left(r^{\prime}\right)^{2} \mathrm{~d} r^{\prime}=\frac{2 M}{\pi}\left(\tan ^{-1} \frac{r}{\sqrt{\theta}}-\frac{r \sqrt{\theta}}{r^{2}+\theta}\right)
$$

which can also be obtained from Equation (3) (Equations (4) and (5) also yield $p_{r}$ and $p_{t}$, as in Ref. [1], but are not needed for present purposes). Due to the smearing, the mass of the particle depends on $\theta$, as one would expect. As in the case of the Gaussian model, the mass of the particle is zero at the center and rapidly rises to $M$. So from a distance, the smearing is no longer apparent and we get an ordinary particle. In other words,

$$
\lim _{\theta \rightarrow 0} M_{\theta}=M
$$

so that the modified Schwarzschild solution reduces to the ordinary Schwarzschild solution (see Figure 1).

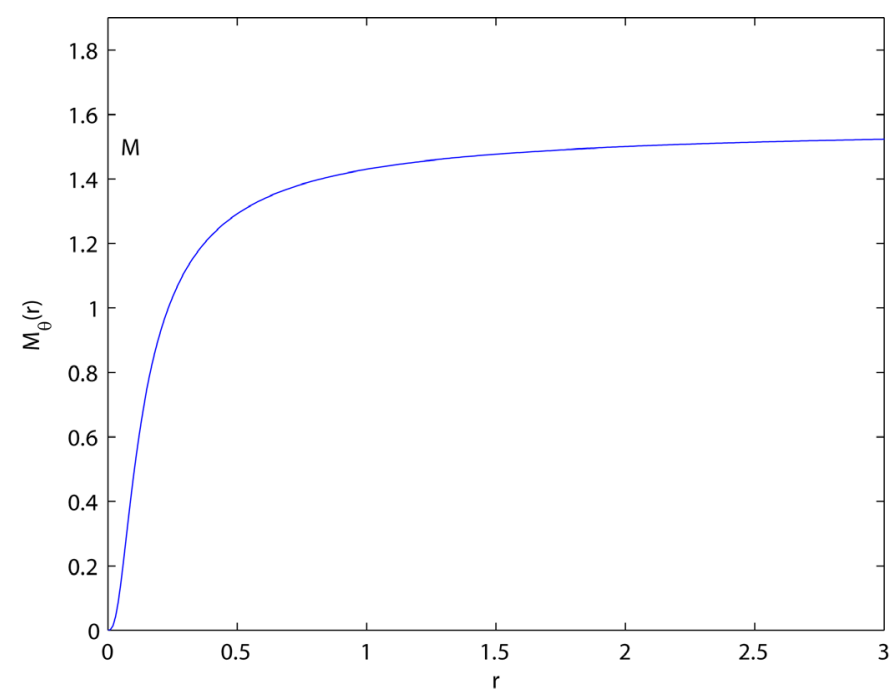

Figure 1. The graph of the smeared mass $M_{\theta}(r)$. 
The mass $M$ could be a diffused centralized object. Since we are interested in galactic rotation curves at some fixed distance $r=R_{0}$ from the center, we will consider instead a thin spherical shell of radius $r=R_{0}$. So instead of a smeared object, we have a smeared spherical surface. We consider the smearing in the outward radial direction only, that being the analogue of the smeared particle at the origin. It follows that $\rho(r)$ in Equation (1) must be replaced by the translated function

$$
\rho(r)=\frac{M \sqrt{\theta}}{\pi^{2}\left[\left(r-R_{0}\right)^{2}+\theta\right]^{2}}
$$

Observe that the mass of the shell becomes

$$
m(r)=\frac{2 M}{\pi}\left[\tan ^{-1} \frac{r-R_{0}}{\sqrt{\theta}}-\frac{\left(r-R_{0}\right) \sqrt{\theta}}{\left(r-R_{0}\right)^{2}+\theta}\right]
$$

again dependent on $\theta$ (Figure 2). Also analogous is $\lim _{\theta \rightarrow 0} m(r)=M$, where $M$ is now the mass of the shell. So in geometrized units, $M$ and $m(r)$ are much less than $R_{0}$.

At this point we can finally address the question of stability by examining the potential $V(r)$ more closely. In view of line element (13), we now have

So from Equation (8),

$$
\mathrm{e}^{-\lambda}=1-\frac{2 m(r)}{r}
$$

$$
V(r)=-E^{2}+E^{2} \frac{r^{-l}}{B_{0}}\left(1-\frac{2 m}{r}\right)-\left(1-\frac{2 m}{r}\right)\left(1+\frac{L^{2}}{r^{2}}\right)
$$

To see the effect of the smearing, we first compute $V^{\prime \prime}(r)$ :

$$
\begin{aligned}
V^{\prime \prime}(r)= & \frac{2 R_{0}^{2} l(l+1)}{2-l} \frac{1}{r^{l+2}}\left(1-\frac{2 m}{r}\right)-\frac{6 l R_{0}^{2}}{2-l} \frac{1}{r^{4}}\left(1-\frac{2 m}{r}\right)+\frac{8 l R_{0}^{2}}{2-l} \frac{1}{r^{l+1}} \frac{r m^{\prime}-m}{r^{2}}-\frac{8 l R_{0}^{2}}{2-l} \frac{1}{r^{3}} \frac{r m^{\prime}-m}{r^{2}} \\
& -\frac{4 R_{0}^{l}}{2-l} \frac{1}{r^{l}} \frac{r^{2} m^{\prime \prime}-2 r m^{\prime}+2 m}{r^{3}}+2\left(1+\frac{l R_{0}^{2}}{2-l} \frac{1}{r^{2}}\right) \frac{r^{2} m^{\prime \prime}-2 r m^{\prime}+2 m}{r^{3}} .
\end{aligned}
$$

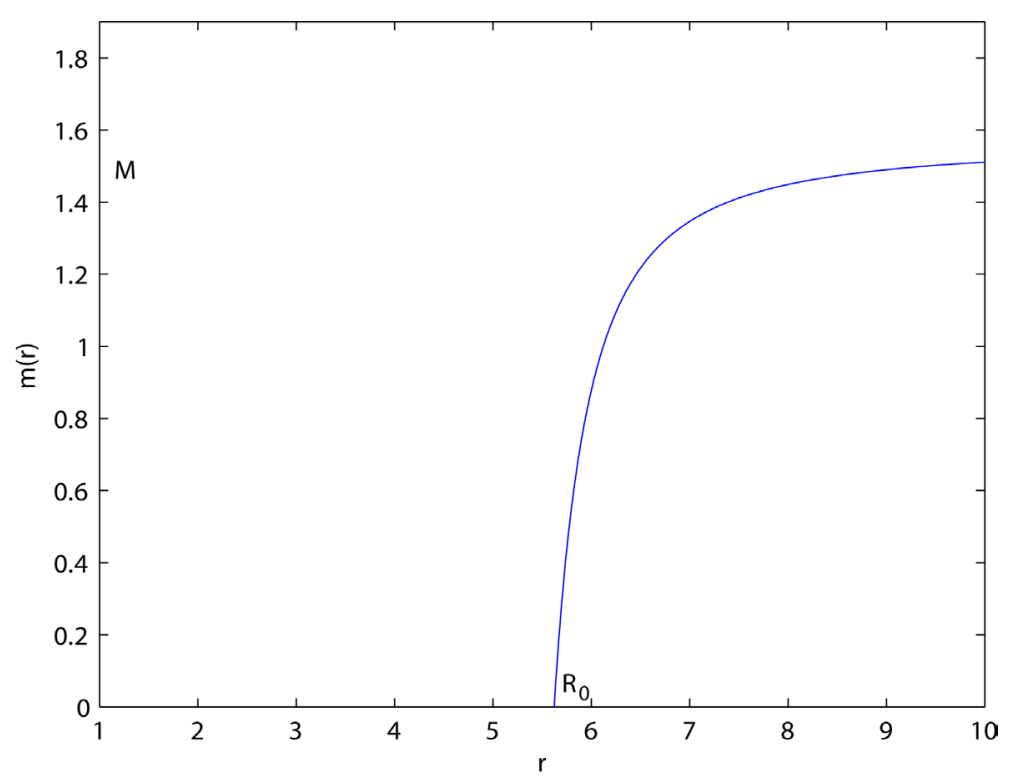

Figure 2. The graph of $m(r)$. 
From Equation (17),

$$
m^{\prime}(r)=\frac{4 M}{\pi} \frac{\left(r-R_{0}\right)^{2} \sqrt{\theta}}{\left[\left(r-R_{0}\right)^{2}+\theta\right]^{2}}
$$

and

$$
m^{\prime \prime}(r)=\frac{8 M}{\pi} \frac{\left(r-R_{0}\right) \sqrt{\theta}-\left(r-R_{0}\right)^{3} \sqrt{\theta}}{\left[\left(r-R_{0}\right)^{2}+\theta\right]^{3}}
$$

It now follows directly that at $r=R_{0}$, only the first two terms in Equation (20) are nonzero:

$$
V^{\prime \prime}\left(R_{0}\right)=\frac{2 R_{0}^{l} l(l+1)}{2-l} \frac{1}{R_{0}^{l+2}}-\frac{6 l R_{0}^{2}}{2-l} \frac{1}{R_{0}^{4}} \approx-\frac{4 l}{(2-l) R_{0}^{2}}<0
$$

We therefore have a stable orbit at $r=R_{0}$ due to the noncommutative geometry.

\section{The Need for Noncommutative Geometry}

We saw in the previous section that the smearing effect in noncommutative geometry is responsible for the stable orbit at $r=R_{0}$. In this section we study the effect of reduced smearing (due to diminishing $\theta$ ), thereby approaching Einstein gravity. The idea is to show that in this limit, the stability criterion is no longer met.

To this end, we return to Equation (20) and observe that the third term,

$$
z(r, \theta)=\frac{8 l R_{0}^{2}}{2-l} \frac{1}{r^{l+1}} \frac{r m^{\prime}-m}{r^{2}}
$$

strongly dominates near $r=R_{0}$ since the denominator is much smaller than the denominator in all the other terms. As we saw, at $r=R_{0}$, both $m^{\prime}$ and $m$ are equal to zero, but $r m^{\prime}-m$ is positive for $r>R_{0}$ and, as we will see later in Figure 3, sharply increasing near $r=R_{0}$ for any fixed $\theta$. So the positive third term easily catches up with the (negative) sum of the first two terms. Given that the remaining terms are negligible, we can now say that there exists an $r=r_{1}$ (for every $\theta$ ) such that

$$
z(r, \theta)-\left.\frac{4 l}{(2-l) R_{0}^{2}}\right|_{r=r_{1}}=0
$$

Hence $V^{\prime \prime}\left(r_{1}\right)=0$ and (for every $\left.\theta\right), V^{\prime \prime}(r)<0$ in the interval $\left[R_{0}, r_{1}\right]$ and $V^{\prime \prime}(r)>0$ for $r>r_{1}$. These cases will be discussed separately.

\section{1. $V^{\prime \prime}(r)<0$}

As noted above, for $r<r_{1}$, we have $V^{\prime \prime}(r)<0$, where $\theta$ is assumed fixed. We wish to show that an ever smaller $\theta$ results in an ever smalller interval $\left[R_{0}, r\right]$ for which $V^{\prime \prime}(r)<0$.

To this end, we obtain from Equation (25),

$$
\frac{8 l R_{0}^{2}}{(2-l) r^{l+1}} \frac{r m^{\prime}-m}{r^{2}}-\left.\frac{4 l}{(2-l) R_{0}^{2}}\right|_{r=r_{1}}=0
$$

and hence from Equations (21) and (22),

$$
\frac{2 M}{\pi} \frac{8 l R_{0}^{2}}{(2-l) r^{l+1}}\left[\frac{2 r\left(r-R_{0}\right)^{2} \sqrt{\theta}}{\left[\left(r-R_{0}\right)^{2}+\theta\right]^{2}}-\tan ^{-1} \frac{r-R_{0}}{\sqrt{\theta}}+\frac{\left(r-R_{0}\right) \sqrt{\theta}}{\left(r-R_{0}\right)^{2}+\theta}\right]-\left.\frac{4 l}{(2-l) R_{0}^{2}}\right|_{r=r_{1}}=0
$$




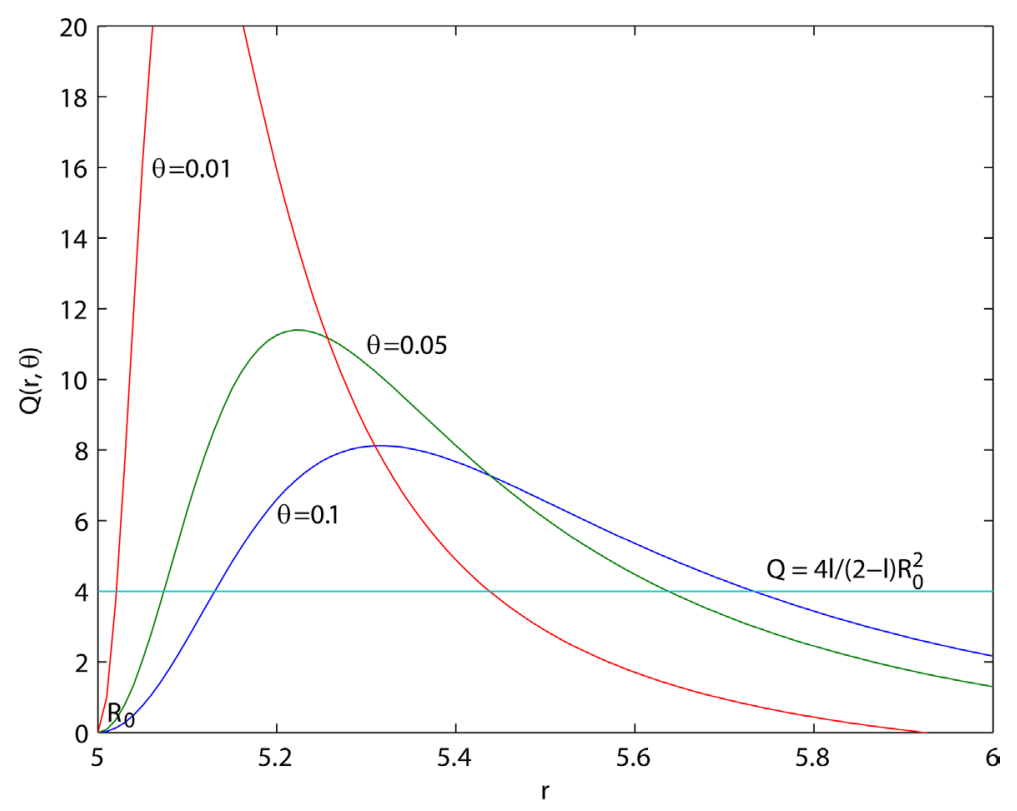

Figure 3. Plot showing that if $\theta \rightarrow 0$, then $r_{1} \rightarrow R_{0}$.

for every fixed $\theta$. So to study the relationship between $\theta$ and $r$ qualitatively, we can choose an arbitrary ray and consider the first term in Equation (27),

$$
Q(r, \theta)=\frac{2 M}{\pi} \frac{8 l R_{0}^{2}}{(2-l) r^{l+1}} \times\left[\frac{2 r\left(r-R_{0}\right)^{2} \sqrt{\theta}}{\left[\left(r-R_{0}\right)^{2}+\theta\right]^{2}}-\tan ^{-1} \frac{r-R_{0}}{\sqrt{\theta}}+\frac{\left(r-R_{0}\right) \sqrt{\theta}}{\left(r-R_{0}\right)^{2}+\theta}\right]
$$

as a function of $r$ and $\theta$ in rectangular coordinates. The condition in Equation (27) can now be viewed as the plane $Q=4 l /(2-l) R_{0}^{2}$ passing through the surface $Q=Q(r, \theta)$. The resulting relationship between $\theta$ and $r$ in this plane is not a simple one-to-one correspondence because the intersection is oval-shaped. However, we know that for physical reasons, $\theta$ is necessarily small and $r$ close to $r=R_{0}$.

So, as a next step, we plot $Q(r, \theta)$ in Equation (28) for a few values of $\theta$, intersected by the line $Q=4 l /(2-l) R_{0}^{2}$, shown in Figure 3. For each curve, the intersection is at $r=r_{1}$. As already noted, there are indeed two values of $r$ for every fixed $\theta$, but only the smaller value is physically relevant. Figure 3 shows that if $\theta \rightarrow 0$, then the left side of Equation (28) can remain fixed only if $r_{1} \rightarrow R_{0}$. By continuity, then, $V^{\prime \prime}(r) \geq 0$ for $r \geq R_{0}$, i.e., the stability criterion is no longer satisfied. We conclude that noncommutative geometry is not only sufficient but also necessary for meeting the stability criterion. Without the noncommutative-geometry background, the stability of the orbit would have to be attributed to another cause, such as dark matter.

\section{2. $V^{\prime \prime}(r)>0$}

Recall that $V^{\prime \prime}(r)>0$ for $r>r_{1}$, for any fixed $\theta$. So outside the smeared region, the stability criterion is no longer met, even though we have a stable orbit at $r=R_{0}$. The implication is that from a distance, the smearing is no longer apparent, even though it is still very much present. So, in a sense, the unseen dark matter is replaced by the unseen noncommutative geometry.

\section{Conclusions}

It is shown in Ref. [1] that a noncommutative-geometry background can account for galactic rotation curves without the need for dark matter. The smearing effect that characterizes noncommutative geometry is described by means of a Gaussian distribution of minimal length $\sqrt{\theta}$. The purpose of this paper is two-fold: 1 ) to confirm 
the conclusions in Ref. [1] in a simpler and more intuitive way by using the distribution proposed in Ref. [8] instead of the Gaussian function, and 2) to show that the smearing effect is both a necessary and sufficient condition for meeting the stability criterion.

That noncommutative geometry, which has all the appearances of a small effect and can account for the galactic rotation curves, is consistent with the corresponding situation in $f(R)$ gravity: only a small change in the Ricci scalar is required to account for dark matter [10].

\section{References}

[1] Rahaman, F., Kuhfittig, P.K.F., Chakraborty, K., Usmani, A.A. and Ray, S. (2012) General Relativity and Gravitation, 44, 905-916. http://dx.doi.org/10.1007/s10714-011-1320-5

[2] Witten, E. (1996) Nuclear Physics B, 460, 335-350. http://dx.doi.org/10.1016/0550-3213(95)00610-9

[3] Seiberg, N. and Witten, E. (1999) Journal of High Energy Physics, 9909, Article ID: 032.

[4] Smailagic, A. and Spalluci, E. (2003) Journal of Physics A, 36, L-467-L-471. http://dx.doi.org/10.1088/0305-4470/36/33/101

[5] Smailagic, A. and Spalluci, E. (2003) Journal of Physics A, 36, L-517-L-521. http://dx.doi.org/10.1088/0305-4470/36/39/103

[6] Nicollini, P., Smailagic, A. and Spalluci, E. (2006) Physics Letters B, 632, 547-551. http://dx.doi.org/10.1016/j.physletb.2005.11.004

[7] Kuhfittig, P.K.F. (2013) International Journal of Pure and Applied Mathematics, 89, 401-408. http://dx.doi.org/10.12732/ijpam.v89i3.11

[8] Nozari, K. and Mehdipour, S.H. (2008) Classical and Quantum Gravity, 25, Article ID: 175015. http://dx.doi.org/10.1088/0264-9381/25/17/175015

[9] Kuhfittig, P.K.F. (Submitted) Macroscopic Traversable Wormholes with Zero Tidal Forces Inspired by Noncommutative Geometry.

[10] Böhmer, C.G., Harko, T. and Lobo, F.S.N. (2008) Astroparticle Physics, 29, 386-392. http://dx.doi.org/10.1016/j.astropartphys.2008.04.003

[11] Nandi, K.K., Valitov, I. and Migranov, N.G. (2009) Physical Review D, 80, Article ID: 047301.

[12] Matos, T., Guzman, F.S. and Nunez, D. (2000) Physical Review D, 62, Article ID: 061301R.

[13] Nandi, K.K., Filippov, A.I., Rahaman, F., Ray, S., Usmani, A.A., Kalam, M. and De Benedictis, A. (2009) Monthly Notices of the Royal Astronomical Society, 399, 2079-2087. http://dx.doi.org/10.1111/j.1365-2966.2009.15399.x 
Scientific Research Publishing (SCIRP) is one of the largest Open Access journal publishers. It is currently publishing more than 200 open access, online, peer-reviewed journals covering a wide range of academic disciplines. SCIRP serves the worldwide academic communities and contributes to the progress and application of science with its publication.

Other selected journals from SCIRP are listed as below. Submit your manuscript to us via either submit@scirp.org or Online Submission Portal.
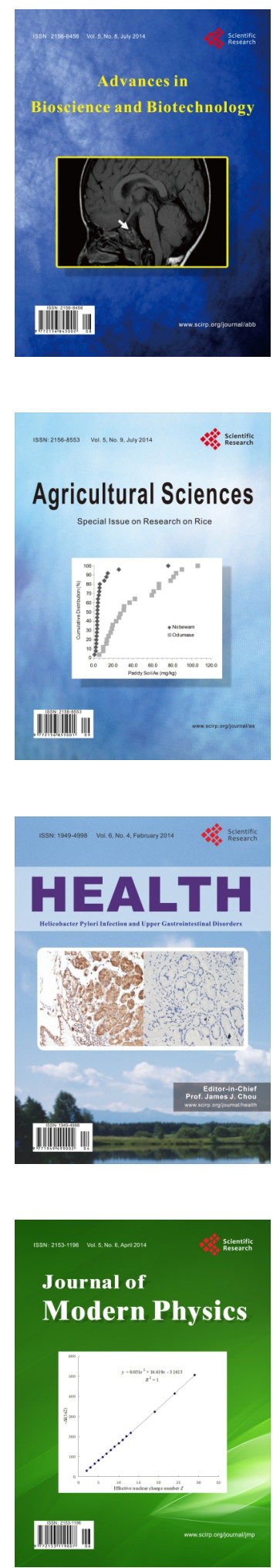
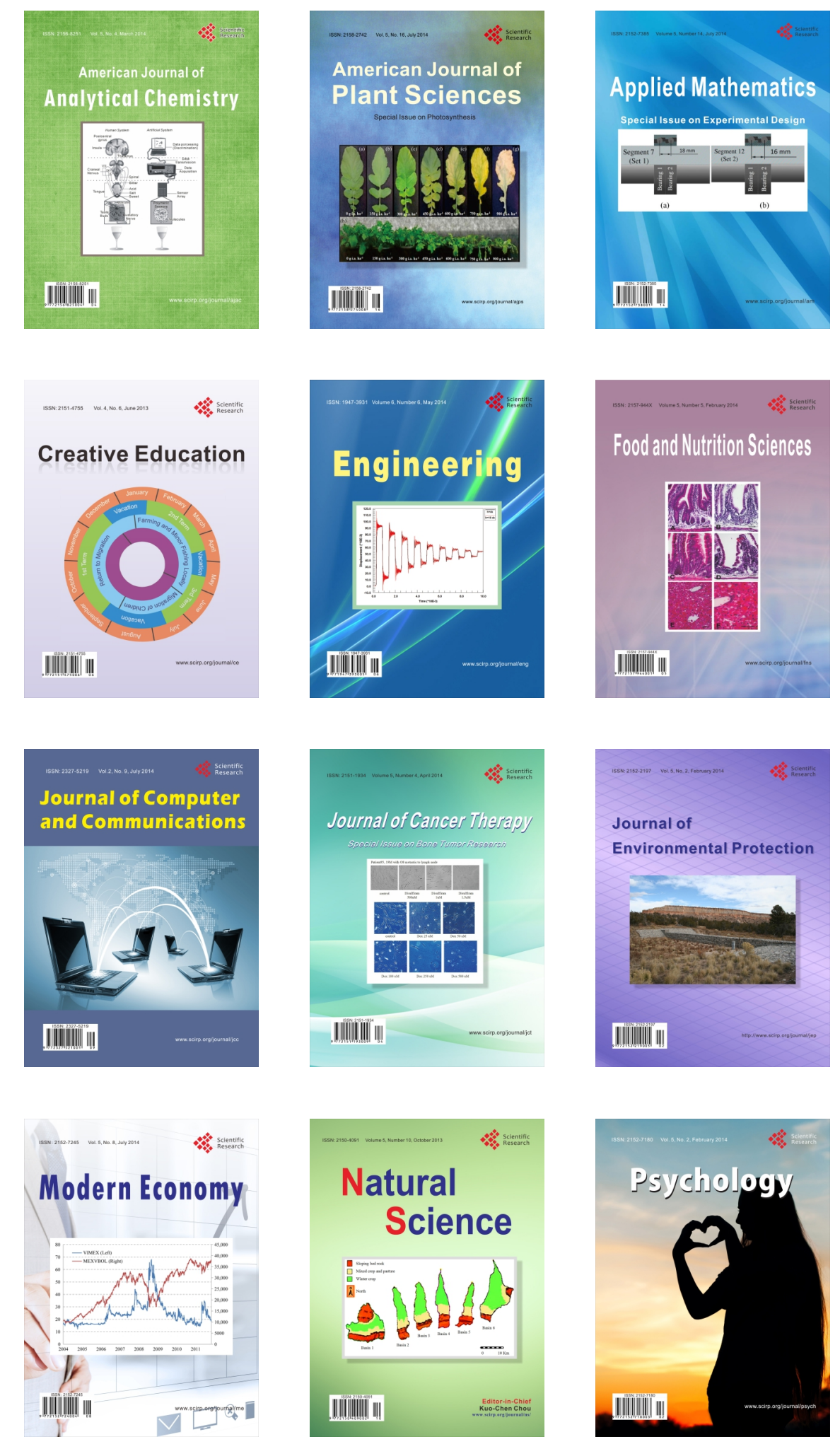